\title{
Study on Electron Distributions in a Three Dimensional Particle Simulation of an Ion Engine
}

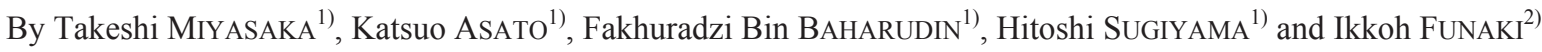 \\ ${ }^{1)}$ Department of Human and Information Systems, Gifu University, Gifu, Japan \\ ${ }^{2)}$ Institute of space and Astronautical Science, Japan Aerospace and Exploration Agency, Kanagawa, Japan
}

(Received June 27th, 2011)

\begin{abstract}
Lifetime evaluations by the numerical analysis of ion engines has become important for long-term missions. To use numerical analysis as a tool for evaluating the engine lifetime, a code that predicts grid erosion caused by the impact of ions quantitatively is required. In most codes, the electron number density is estimated from the Boltzmann relation because the calculation time of three-dimensional analyses becomes huge. In this paper, to evaluate the applicability of the Boltzmann relation in ion engine analysis, three-dimensional analyses of a Hayabusa type three-grid ion engine using a full-PIC (particle in cell) code and hybrid-PIC code using the Boltzmann relation were performed. The comparison of the results revealed that the electron number densities obtained by the two analyses agree well in almost the entire region. However, in the downstream neutralizing region, where the electric potential is positive, the electron number density for the hybrid-PIC code increases up to about seven times higher than that for the full-PIC code.
\end{abstract}

Key Words: Electric Propulsion, Ion Engine, PIC Code, Boltzmann Relation, Grid Erosion

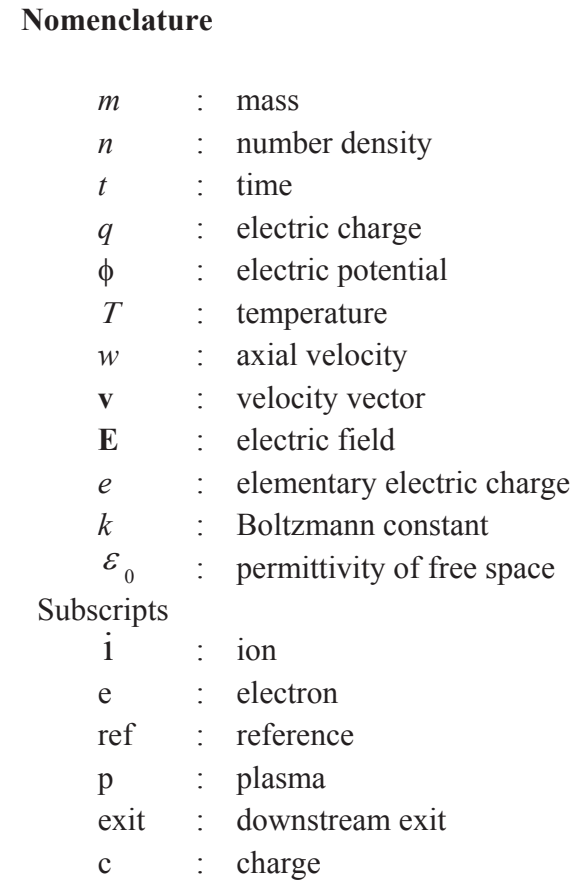

\section{Introduction}

Since an ion engine was used as the main engine of the asteroid probe Hayabusa, there has been high expectations for long-term missions ${ }^{1,2)}$. In the past, experimental and numerical studies of ion engines were performed labor-intensively ${ }^{1-13)}$. One of the factors that has become important in the development of ion engines for long-term missions is the difficulty of lifetime evaluation by experiments ${ }^{2}$. Thus, evaluation of the lifetime by numerical analysis has become important. For lifetime evaluations, investigations of the models employed for numerical analyses through quantitative evaluations are required.

In previous numerical analyses, various types of models have been employed. The flux tube model has been used for describing an ion beam. The flux tube model treats ion particle motions as ion fluxes and reduces the calculation cost. To estimate the electron number distribution, the Boltzmann relation has been employed for most previous numerical codes. The electron motion of ion engines having a very small mass using multihole grids results in an enormous calculation time. By evaluating the electron number density distributions using the Boltzmann relation, ion engine properties can be obtained with a significantly reduced calculation time.

However, because the electron number density affects the electric potential distribution through the Poisson equation, validation of the applicability of the Boltzmann relation to quantitative analyses, such as lifetime evaluation, is required.

In the present study, analyses of a three-grid ion engine by a hybrid-PIC (particle in cell) code ${ }^{14)}$ using the Boltzmann relation and a full-PIC code, which treats electrons as particles, were performed. By a comparison between the results obtained by the two codes with different electron models, the effects of the electron model on electron number density distributions were investigated. The electron number density obtained by the full-PIC code was examined to check whether it satisfies the Boltzmann relation for the electric potential. The Maxwell distribution is a precondition for satisfying the Boltzmann relation. Thus, electron velocity distributions obtained by the full-PIC code were also investigated. On the basis of the above examination, the differences in the electron number density and electric potential distributions obtained by full-PIC and hybrid-PIC analyses were studied, and the 
applicability of the Boltzmann relation to the electron number density distribution in ion engines was validated.

\section{Numerical Method}

\subsection{Procedures}

The present analyses were carried out using two different codes, the full-PIC and hybrid-PIC codes. Figure 1 shows the numerical procedures of the two codes. The specific procedures and equations involved the codes are explained in detail below.

i) First, the motion of neutral particles is calculated as a preparatory analysis. The calculated neutral number density distribution is used for calculations of collisions between ions and neutrals.

After the initial conditions are given, the following calculations of charged particles are performed.

ii) The motion of ion particles under a electric field is calculated by Eq. (1).

$$
m_{\mathrm{i}} \frac{d \mathbf{v}_{\mathrm{i}}}{d t}=e \mathbf{E}
$$

Ion number densities at grid points are estimated by weighting the calculated ion particle positions. The electric potential E in Eq. (1) is obtained by weighting the electric fields at the surrounding grid points. These weightings are carried out by a PIC method.

The treatment of electron particle motion is different in the two codes.

iii) In full-PIC analysis, as for the ion particle motion, electron particle motion is calculated under an electric field by Eq. (2). The electron number densities at grid points are estimated by weighting the calculated electron particle positions.

$$
m_{\mathrm{e}} \frac{d \mathbf{v}_{\mathrm{e}}}{d t}=e \mathbf{E}
$$

In past work, ${ }^{11)}$ it was found that for a ratio of ion mass to electron mass of higher than 10000 , the electron distribution was nearly independent of the ratio. Thus, to shorten the calculation time, an artificial electron mass, assumed to be 10000 times smaller than the ion mass, is introduced in the calculation.

iv) In hybrid-PIC analysis, the electron number density at each grid point is directly obtained by substituting the electric potential into the following Boltzmann relation.

$$
n_{\mathrm{e}}=n_{\mathrm{e}, \text { ref }} \exp \left[\frac{e\left(\phi-\phi_{\mathrm{ref}}\right)}{k T_{\mathrm{e}}}\right]
$$

v) The charge-exchange and elastic collisions between ions and neutrals are next calculated. Trajectory tracking of the ions and neutrals after collision is also carried out.

vi) Using the charge density distribution obtained from steps ii)-iv), electric potential distributions are then obtained by solving the Poisson equation.

$$
\Delta \phi=-\frac{\rho_{\mathrm{c}}}{\varepsilon_{0}}
$$

The Poisson equation is solved by the FEM (finite element method) using linear tetrahedral elements. In hybrid-PIC analysis, to obtain the steady electric potential distribution, the simultaneous equations Eqs. (3) and (4) must be solved. Thus, iterative calculations of these equations are required. Electric field distributions are obtained from the electric potential distribution.

The calculations in steps ii)-v) are repeated to obtain convergent distributions. After the distributions have converged, the data are saved.

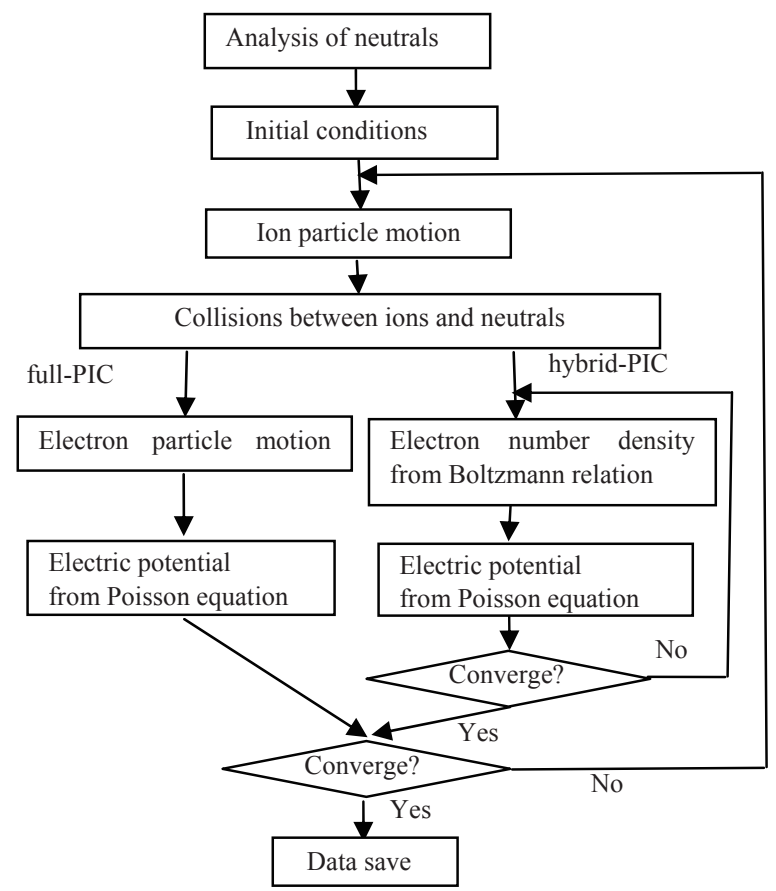

Fig. 1. Numerical procedures.

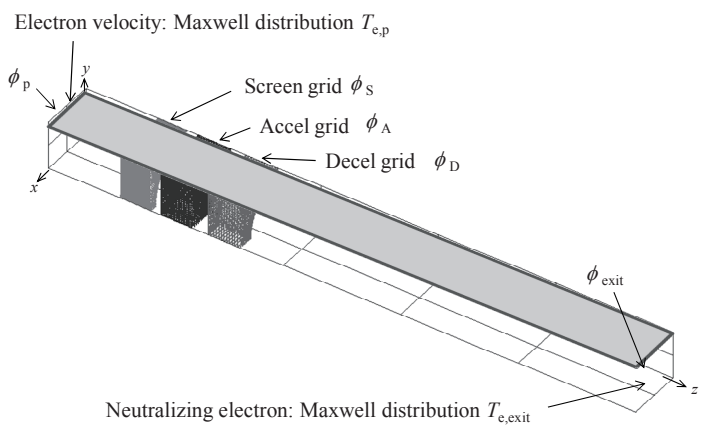

Fig. 2. Schematic of three-dimensional calculation domain and boundary conditions.

\subsection{Numerical domain and conditions}

A schematic of the calculation domain of the three-grid ion engine is shown in Fig. 2. The geometric parameters of the ion engine are set as close as possible to those of the Hayabusa $\mu 10$ engine. To reduce the calculation time, a calculation domain having a quarter hole with symmetric boundary 
conditions is used. In the present analyses, a rectangular grid is employed. The numbers of grid points are 43, 39, and 170 in the $x$-, $y$-, and $z$-directions, respectively. The grid size is not uniform in the $z$-direction. In the discharge and neutralizing regions, the grid sizes are determined on the basis of the respective Debye lengths.

The fixed electric potentials are given in Table 1 . At the upstream boundary, ions with nonzero axial velocity and electrons with Maxwell velocities flow into the calculation domain. Neutralizing electrons also flow into the domain at the downstream boundary.

\begin{tabular}{|l|r|} 
Table 1. Calculation conditions. \\
\begin{tabular}{|l|r|}
\hline propellant & xenon \\
\hline plasma electric potential, $\phi_{\mathrm{p}}$ & $1510 \mathrm{~V}$ \\
\hline screen grid electric potential, $\phi_{\mathrm{S}}$ & $1500 \mathrm{~V}$ \\
\hline accel grid electric potential, $\phi_{\mathrm{A}}$ & $-350 \mathrm{~V}$ \\
\hline decel grid electric potential, $\phi_{\mathrm{D}}$ & $0 \mathrm{~V}$ \\
\hline electric potential at exit, $\phi_{\text {exit }}$ & $0 \mathrm{~V}$ \\
\hline
\end{tabular}
\end{tabular}

\section{Results and Discussion}

The electric potential distributions obtained by full-PIC and hybrid-PIC analyses are shown in Fig. 3. There is a region of positive electric potential in the vicinity of the hole center of the decel grid due to the existence of a converged ion beam. The effects of the electron model on the electron number density at two points (A) and (B) shown in Fig. 3 were investigated.

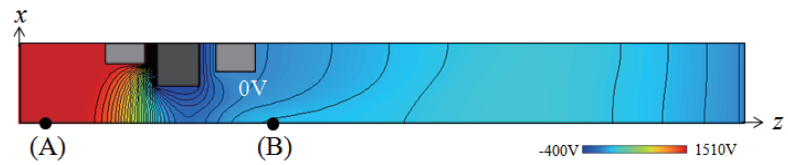

(a) Full-PIC

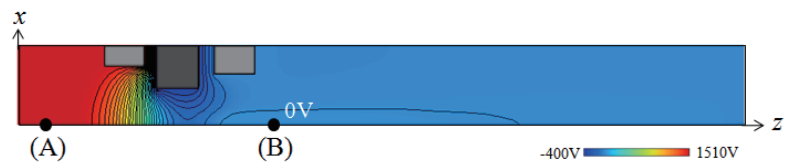

(b) Hybrid-PIC

Fig. 3. Two-dimensional distribution of electric potential $(y=0 \mathrm{~mm})$.

Figure 4 shows the electron number densities (A) in the pre-sheath region and (B) in the vicinity of the hole center of the decel grid calculated by full-PIC analysis. In addition, to confirm that these densities satisfy the Boltzmann relation, electron number densities estimated by substituting the relative electric potential calculated by full-PIC analysis into the Boltzmann relation are also shown in Fig. 4. In the case of estimation using the Boltzmann relation at (A), the electric potential and electron number density at the upstream boundary are used as reference values in Eq. (3). Because the electrons at (B) are neutralizing electrons flowing from the downstream, for the estimation at (B), the electric potential and electron number density in the downstream are used as reference values. In Fig. 4, the electron number density at (A) obtained by full-PIC analysis agree well with those estimated using the Boltzmann relation. However, the electron number densities at (B) are different; the estimated number density is higher than that obtained by full-PIC analysis. While the electric potential at (A) is less than the reference value as shown in Fig. 3, the electric potential at (B) is a positive value, that is, higher than the reference value of $0 \mathrm{~V}$.

One of the preconditions for satisfying the Boltzmann relation is that the electrons have the following Maxwell distribution $f$.

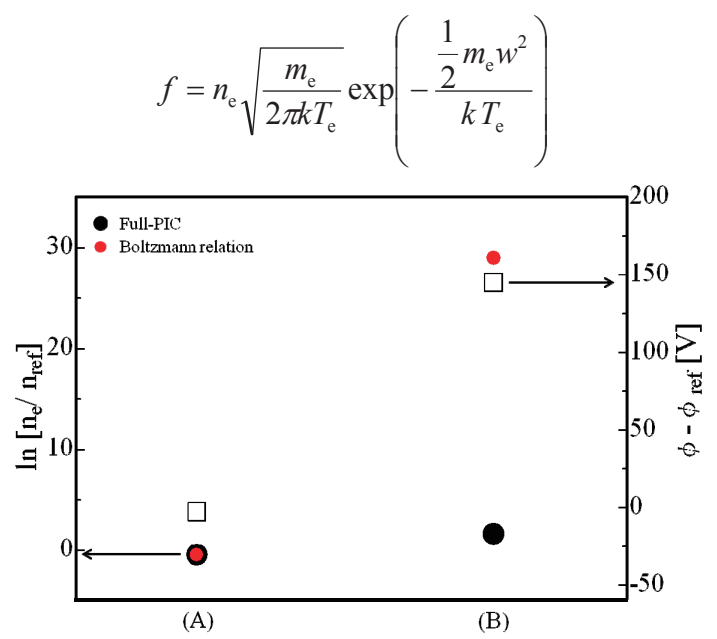

Fig. 4. The electron number density and electric potential difference from the relative reference value obtained by full-PIC analysis at (A) and (B) and estimated electron number density obtained by substituting the relative electric potential obtained by full-PIC analysis into the Boltzmann relation.

To investigate the results of comparing the electron number densities in Fig. 4, the axial velocity distributions at (A) and (B) are shown in Fig. 5. At (A), where the electron number density clearly satisfy the Boltzmann relation, these axial velocity distributions agree well with the corresponding Maxwell distributions. However, the axial velocity distribution at (B) differs from the Maxwell distribution and contains a higher proportion of electrons having high speed compared with the Maxwell distribution. The high-speed part of the distribution satisfies the velocity distribution $g$ given by Eq. (6), which involves the electric potential difference from the reference value:

$$
g=n_{\mathrm{e}, \text { ref }} \sqrt{\frac{m_{\mathrm{e}}}{2 \pi k T_{\mathrm{e}}}} \exp \left[-\frac{\frac{1}{2} m_{\mathrm{e}} w^{2}-e\left(\phi-\phi_{\text {ref }}\right)}{k T_{\mathrm{e}}}\right]
$$

However, the proportion of electrons having a low speed is lower than that calculated by Eq. (6). This low number causes the difference between the electron number density at (B) and that estimated using the Boltzmann relation shown in Fig. 4.

The reason for the difference in the axial velocity distribution at (B) is that the positive electric potential at (B) is higher than the reference value of $0 \mathrm{~V}$ at the downstream boundary where the electrons flow with the Maxwell 
distribution. With increasing electric potential, the velocities of the electrons in the entire axial velocity region increase and the number of electrons with a low speed decreases. The reason why electrons with a low speed are observed in the case of full-PIC analysis is the existence of the electrons with nonzero radial velocity components.

The results of investigating the axial velocity distribution indicate that the Boltzmann relation is inapplicable for the evaluation of electrons in the region where the electric potential is higher than the reference value.

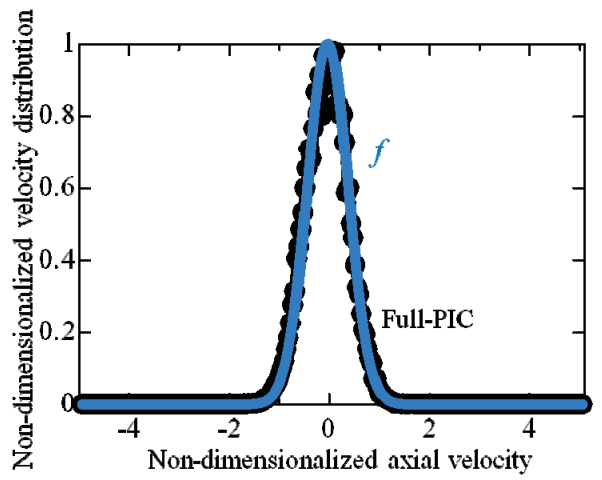

(a) Distributions at (A)

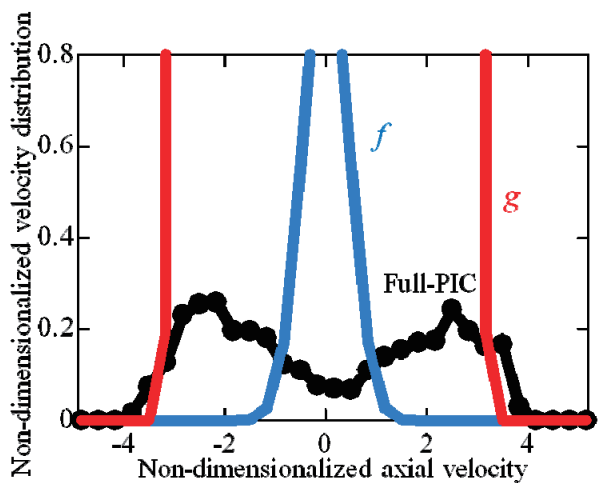

(b) Distributions at (B)

Fig. 5. Axial velocity distributions of electrons at (A) and (B) and the Maxwell distributions for the relative electric potential. The axial velocity and the velocity distributions are non-dimensionalized by the velocity $\sqrt{2 \pi k T_{\mathrm{e}} / m_{\mathrm{e}}}$ and the peak value of $f$, respectively.

Figure 6 shows the electron number densities at (B) obtained by hybrid-PIC analysis, full-PIC analysis, and estimation using the Boltzmann relation with the electric potential obtained by full-PIC analysis. While the number density obtained by hybrid-PIC analysis is higher than that obtained by full-PIC analysis, it is lower than the density estimated using the electric potential obtained by full-PIC analysis. In hybrid-PIC analysis, since the increase in the electron number density at (B) causes a decrease in the electric potential, the difference between the electric potential and the reference value becomes smaller. As a result, the electron number density obtained by hybrid-PIC analysis approaches the value obtained by full-PIC analysis.

Figure 7 shows the two-dimensional distributions of electron number density obtained by full-PIC and hybrid-PIC analyses. In Fig. 7, the difference shown in Fig. 6 is clearly observed. In the vicinity of the hole center of the decel grid where the electric potential is higher than the reference value, the electron number density obtained by full-PIC analysis is lower than that obtained by hybrid-PIC analysis. The difference between the electron number densities causes the difference between the electric potential distributions shown in Fig. 3. The electric potential distribution influences trajectories of ions impacting on the grids in evaluations of grid erosions. Thus, investigations of the applicability of the Boltzmann relation in the neutralizing region are required under a wide range of operating conditions.

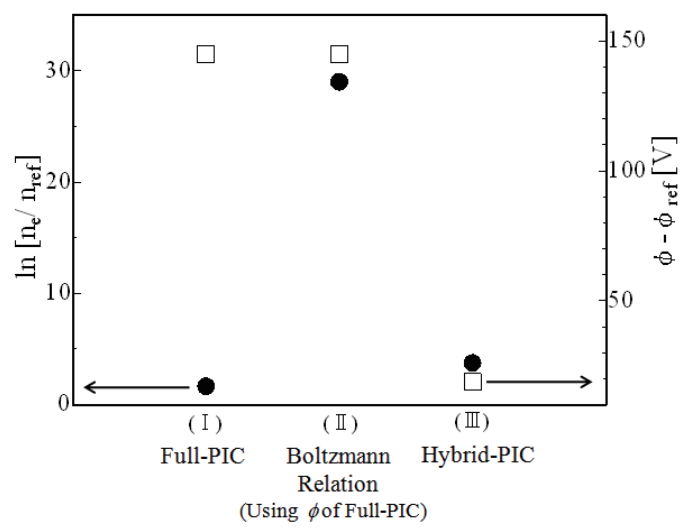

Fig. 6. Electron number densities and electric potentials calculated by (I) full-PIC and (III) hybrid-PIC analyses at (B) and (II) electron number density estimated by substituting the electric potential obtained by full-PIC analysis into the Boltzmann relation.

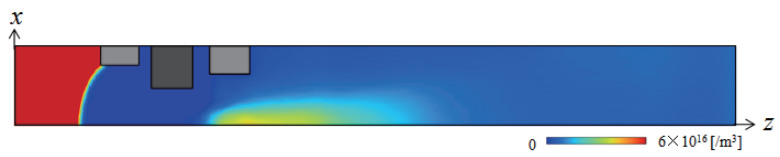

(a) Full-PIC

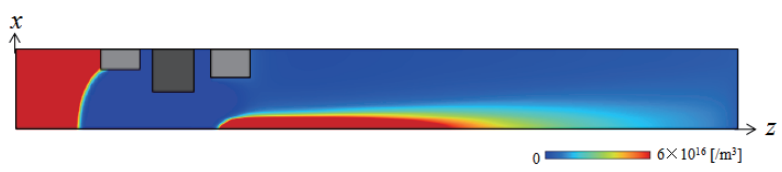

(b) Hybrid-PIC

Fig. 7. Two-dimensional distributions of electron number density calculated by full-PIC and hybrid-PIC analyses.

\section{Conclusion}

To evaluate the effects of the electron model on the electron number density in three-grid ion engines, three-dimensional analyses were performed using the full-PIC code, treating electrons as particles, and hybrid-PIC code, using the Boltzmann relation for the calculation of electron number density. The results of full-PIC analysis show that the electron number density clearly satisfies the Boltzmann relation with the calculated electric potential in the upstream region. However, in the area of positive electric potential in the neutralizing region, the electron number density is lower 
than the Boltzmann relation. By investigating electron axial velocity distributions, it was found that the non-Maxwell distribution of the axial velocity in the region of positive electric potential caused the deviation from the Boltzmann relation. The electron number density obtained by hybrid-PIC analysis is closer to that obtained by full-PIC analysis than the estimated value obtained using the Boltzmann relation with the electric potential obtained from full-PIC analysis.

The comparison of the results for the two different electron models revealed that the applicability of the Boltzmann relation to the calculation of electron number density is valid in the upstream region. However, the present results also indicate that for detailed quantitative analysis, an investigation of the applicability of the Boltzmann relation to the calculation of neutralizing electrons is required.

\section{Acknowledgments}

This work was supported by Japan Aerospace Exploration Agency under cooperative research. The authors would like to thank members of the JIEDI project for their supports.

\section{References}

1) Funaki, I., Kuninaka, H. and Toki, K.: Plasma Characterization of a 10-cm Diameter Microwave Discharge Ion Thruster, J. Propulsion and Power, 20 (2004), pp.718-727.

2) Kuninaka, H.: Research and Development of JIEDI (JAXA Ion Engine Development Initiatives) Tool for Numerical Evaluation of Ion Engine Grid Lifetime, JAXA Special Publication, JAXA-SP-06-019, pp.5-9, 2007 (in Japanese).
3) Nakano, M. and Arakawa, Y.: Numerical Simulation of Ion Thruster Grid Lifetime, J. Jpn. Soc. for Aeronaut. Apace Sci, 48 (2000), pp.111-117 (in Japanese).

4) Nakano, M.: Three-dimensional Simulations of Ion Engine Grid Erosion, Advances in Applied Plasma Science, 6 (2007), pp.93-96.

5) Nakano, M.: Improvements in the Grid Erosion Evaluation Code for Ion Engines, JAXA-SP-06-019, JAXA Special Publication, pp.47-53, 2007 (in Japanese).

6) Nakayama, N. and Wilbur, P.J.: Numerical Simulation of Ion Beam Optics for Multiple-grid Systems, J. Propulsion and Power, 19 (2003), pp.607-613.

7) Wang, J., Cao, Y., Kafafy, R., Martinez, R. and Williams, J.: AIAA paper 2006-4999, 2006.

8) Tartz, M., Hartmann, E., Deltschew, R. and Neumann, H.: Validation of a Grid Erosion Simulation by Short-time Erosion Measurements, Proceedings of the 26th International Electric Propulsion Conference, IEPC-99-147, 1999.

9) Okawa, Y.and Takegahara, H.: Particle Simulation on Ion Beam Extraction Phenomena in an Ion Thruster, Proceedings of the 26th International Electric Propulsion Conference, IEPC-99-146, 1999.

10) Miyasaka, T, Kobayashi, T. and Asato, K.: Characteristics of Ions Impacting Grid Surfaces in an Ion Engine, Vacuum, 85(2010), pp.585-590.

11) Miyasaka, T., Kobayashi, T. and Asato, K.: Numerical Prediction of Grid Erosion of Ion Engine, Trans. of the Japan Soc. for Aeronautical and Space Sciences, Aerospace Technology Japan, 8 (2010), pp.Pb 61-Pb 66 .

12) Hayakawa, Y.: Measurements of Current Distribution on a Two-grid-ion-extraction-system Gridlet, Proceedings of 42nd Joint Propulsion Conference, AIAA-2006-5003, pp.1-9, 2006.

13) Hayakawa, Y.: Current Distribution on a Two-grid-ion-extraction-system Gridlet, JAXA Special Publication: JAXA-SP-06-019, pp.20-27, 2007 (in Japanese).

14) Birdsall, C.K. and Langdon, A.B.: Plasma Physics via Computer Simulation, MacGraw-Hill, 1985. 